\title{
Signal and backgrounds for leptoquarks at the CERN LHC. II. Vector leptoquarks
}

\author{
A. Belyaev, ${ }^{1,2, *}$ O. J. P. Éboli, ${ }^{1, \dagger}$ R. Z. Funchal, ${ }^{3, \$}$ and T. L. Lungov ${ }^{1, \S}$ \\ ${ }^{1}$ Instituto de Física Teórica - UNESP, R. Pamplona 145, 01405-900 São Paulo, Brazil \\ ${ }^{2}$ Skobeltsyn Institute of Nuclear Physics, Moscow State University, 119899 Moscow, Russian Federation \\ ${ }^{3}$ Instituto de Física, Universidade de São Paulo, Caixa Postal 66.318, 05315-970 São Paulo, Brazil
}

(Received 5 November 1998; published 4 March 1999)

\begin{abstract}
We perform a detailed analyses of the CERN Large Hadron Collider (LHC) capability to discover first generation vector leptoquarks through their pair production. We study the leptoquark signals and backgrounds that give rise to final states containing a pair $e^{+} e^{-}$and jets. Our results show that the LHC will be able to discover vector leptoquarks with masses up to $1.8-2.3 \mathrm{TeV}$ depending on their couplings to fermions and gluons. [S0556-2821(99)00407-5]

PACS number(s): 12.60.-i, 13.85.Rm, 14.80.-j
\end{abstract}

\section{INTRODUCTION}

In the standard model (SM) the cancellation of chiral anomalies takes place only when we consider the contributions of leptons and quarks, indicating a deeper relation between them. Therefore, it is rather natural to consider extensions of the SM that treat quarks and leptons on the same footing and consequently introduce new bosons, called leptoquarks, which mediate quark-lepton transitions. The class of theories exhibiting these particles includes composite models [1,2], grand unified theories [3], technicolor models [4], and superstring-inspired models [5]. Since leptoquarks couple to a lepton and a quark, they are color triplets under $\mathrm{SU}(3)_{C}$, carry simultaneously lepton and baryon number, have fractional electric charge, and can be of scalar or vector nature.

From the experimental point of view, leptoquarks possess the striking signature of a peak in the invariant mass of a charged lepton with a jet, which make their search much simpler without the need of intricate analyses of several final state topologies. Certainly, the experimental observation of leptoquarks is an undeniable signal of physics beyond the SM; so there have been a large number of direct searches for them in $e^{+} e^{-}$[6], $e^{ \pm} p$ [7], and $p \bar{p}$ [8] colliders. Up to now all of these searches led to negative results, which bound the mass of vector leptoquarks to be larger than 245-340 (230325) $\mathrm{GeV}$, depending on the leptoquark coupling to gluons, for branching ratio into an $e^{ \pm}$and jet equal to $1(0.5)$ [9].

The direct search for leptoquarks with masses above a few hundred $\mathrm{GeV}$ can be carried out only in the next generation of $p p$ [10], ep [11,12], $e^{+} e^{-}[13], e^{-} e^{-}[14]$, e $\gamma$ [15], and $\gamma \gamma$ [16] colliders. In this work, we extend our previous analyses of the CERN Large Hadron Collider (LHC) potentiality to discover scalar leptoquarks to vector ones [17]. We study the pair production of first generation leptoquarks that lead to a final state topology containing two

\footnotetext{
*Email address: belyaev@ift.unesp.br

${ }^{\dagger}$ Email address: eboli@ift.unesp.br

‡Email address: zukanov@charme.if.usp.br

${ }^{\S}$ Email address: lungov@ift.unesp.br
}

jets plus a pair $e^{+} e^{-}$. We analyze this signal for vector leptoquarks and use the results for the SM backgrounds obtained in Ref. [17] where careful studies of all possible top quark production, QCD, and electroweak backgrounds for this topology were performed using the event generator PYTHIA [18]. We restrict ourselves to first generation leptoquarks that couple to pairs $e^{ \pm} u$ and $e^{ \pm} d$ with the leptoquark interactions described by the most general effective Lagrangian invariant under $\mathrm{SU}(3)_{C} \otimes \mathrm{SU}(2)_{L} \otimes \mathrm{U}(1)_{Y}[11]$.

In this work, we study the pair production of vector leptoquarks via quark-quark and gluon-gluon fusions, i.e.

$$
\begin{aligned}
& q+\bar{q} \rightarrow \Phi_{\mathrm{lq}}+\Phi_{\mathrm{lq}}, \\
& g+g \rightarrow \Phi_{\mathrm{lq}}+\bar{\Phi}_{\mathrm{lq}},
\end{aligned}
$$

where we denote the vector leptoquarks by $\Phi_{\text {lq }}$. These processes give rise to $e^{+} e^{-}$pairs with large transverse momenta accompanied by jets. Using the cuts devised in Ref. [17] to reduce the backgrounds and enhance the signals, we show that the LHC will be able to discover first generation vector leptoquarks with masses smaller than 1.5-2.3 TeV, depending on their couplings and on the integrated luminosity (10 or $100 \mathrm{fb}^{-1}$ ).

Here, we perform our analyses using a specially created event generator for vector leptoquarks. Moreover, we consider the most general coupling of vector leptoquarks to gluons, exhibiting our results for two distinct scenarios. In particular we analyze the most conservative case where the leptoquark couplings to gluons is such that the pair production cross section is minimal [26]. While we were preparing this paper, a similar study of the production of vector leptoquarks appeared [19], which uses a different event generator, distinct cuts, and a less general leptoquark coupling to gluons, which contains only the chromomagnetic anomalous coupling to gluons.

Low-energy experiments give rise to strong constraints on leptoquarks, unless their interactions are carefully chosen $[20,21]$. In order to evade the bounds from proton decay, leptoquarks are required not to couple to diquarks. To avoid the appearance of leptoquark induced flavor changing neutral current (FCNC), leptoquarks are assumed to couple only to a single quark family and only one lepton generation. Never- 
theless, there still exist low-energy limits on leptoquarks. Helicity suppressed meson decays restrict the couplings of leptoquarks to fermions to be chiral [20]. Moreover, residual FCNC [22], atomic parity violation [23], effects of leptoquarks on the $Z$ physics through radiative corrections [24], and meson decay $[22,23,25]$ constrain the first generation leptoquarks to be heavier than $0.5-1.5 \mathrm{TeV}$ when the coupling constants to fermions are equal to the electromagnetic coupling $e$. Therefore, our results indicate that the LHC cannot only confirm these indirect limits but also expand them considerably.

The outline of this paper is as follows. In Sec. II we introduce the $\mathrm{SU}(3)_{C} \otimes \mathrm{SU}(2)_{L} \otimes \mathrm{U}(1)_{Y}$ invariant effective Lagrangians that we analyzed. In Sec. III we describe in detail how we have performed the signal Monte Carlo simulation. Section IV contains a brief summary of the backgrounds and kinematical cuts needed to suppress them. Our results and conclusions are shown in Sec. V.

\section{MODELS FOR VECTOR LEPTOQUARK INTERACTIONS}

In this work we assume that leptoquarks decay exclusively into the known quarks and leptons. In order to avoid the low-energy constraints, leptoquarks must interact with a single generation of quarks and leptons with chiral couplings. Furthermore, we also assume that their interactions are $\mathrm{SU}(3)_{C} \otimes \mathrm{SU}(2)_{L} \otimes \mathrm{U}(1)_{Y}$ gauge invariant above the electroweak symmetry breaking scale $v$. The most general effective Lagrangian satisfying these requirements and baryon number $(B)$, lepton number $(L)$, electric charge, and color conservations is [11]

$$
\begin{aligned}
\mathcal{L}_{\mathrm{eff}}^{\mathrm{f}}= & \mathcal{L}_{F=2}+\mathcal{L}_{F=0}+\text { H.c. }, \\
\mathcal{L}_{F=2}= & g_{2 \mathrm{~L}}\left(V_{2 L}^{\mu}\right)^{T} \bar{d}_{R}^{c} \gamma_{\mu} i \tau_{2} \ell_{L}+g_{2 \mathrm{R}} \bar{q}_{L}^{c} \gamma_{\mu} i \tau_{2} e_{R} V_{2 R}^{\mu} \\
& +\tilde{g}_{2 \mathrm{~L}}\left(\widetilde{V}_{2 L}^{\mu}\right)^{T} \bar{u}_{R}^{c} \gamma_{\mu} i \tau_{2} \ell_{L}, \\
\mathcal{L}_{F=0}= & h_{1 \mathrm{~L}} \bar{q}_{L} \gamma_{\mu} \ell_{L} V_{1 L}^{\mu}+h_{1 \mathrm{R}} \bar{d}_{R} \gamma_{\mu} e_{R} V_{1 R}^{\mu} \\
& +\widetilde{h}_{1 \mathrm{R}} \bar{u}_{R} \gamma_{\mu} e_{R} \widetilde{V}_{1 R}^{\mu}+h_{3 \mathrm{~L}} \bar{q}_{L} \vec{\tau} \gamma_{\mu} \ell_{L} \cdot \vec{V}_{3 L}^{\mu},
\end{aligned}
$$

where $F=3 B+L, q(\ell)$ stands for the left-handed quark (lepton) doublet, and $u_{R}, d_{R}$, and $e_{R}$ are the singlet components of the fermions. We denote the charge-conjugated fermion fields by $\psi^{c}=C \bar{\psi}^{T}$ and we omitted in Eqs. (4) and (5) the flavor indices of the leptoquark couplings to fermions. The leptoquarks $V_{1 R(L)}^{\mu}$ and $\widetilde{V}_{1 R}^{\mu}$ are singlets under $\mathrm{SU}(2)_{L}$, while $V_{2 R(L)}^{\mu}$ and $\widetilde{V}_{2 L}^{\mu}$ are doublets, and $V_{3 L}^{\mu}$ is a triplet.

From the above interactions we can see that for first generation leptoquarks, the main decay modes of leptoquarks are those into pairs $e^{ \pm} q$ and $\nu_{e} q^{\prime}$. In this work we do not consider their decays into neutrinos; however, we take into account properly the branching ratio into charged leptons. In Table I we exhibit the leptoquarks that can be studied using the final state $e^{ \pm}$plus a jet, as well as their decay products and branching ratios. Only the leptoquarks $V_{2 L}^{2}, \widetilde{V}_{2 L}^{2}$, and
TABLE I. Vector leptoquarks that can be observed through their decays into a $e^{ \pm}$and a jet and the correspondent branching ratios into this channel.

\begin{tabular}{ccc}
\hline \hline Leptoquark & Decay & Branching ratio \\
\hline$V_{2 L(R)}^{1}$ & $e^{-} d$ & $100 \%$ \\
$V_{2 R}^{2}$ & $e^{-} u$ & $100 \%$ \\
$\widetilde{V}_{2 L}^{1}$ & $e^{-} u$ & $100 \%$ \\
$V_{1 L}$ & $e^{+} d$ & $50 \%$ \\
$V_{1 R}$ & $e^{+} d$ & $100 \%$ \\
$\widetilde{V}_{1 R}$ & $e^{+} u$ & $100 \%$ \\
$V_{3 L}^{-}$ & $e^{+} u$ & $100 \%$ \\
$V_{3 L}^{0}$ & $e^{+} d$ & $50 \%$ \\
\hline \hline
\end{tabular}

$V_{3}^{+}$decay exclusively into a jet and a neutrino, and are not constrained by our analyses; see Eqs. (4) and (5).

Leptoquarks are color triplets; therefore, it is natural to assume that they interact with gluons. However, the $\mathrm{SU}(2)_{C}$ gauge invariance is not enough to determine the interactions between gluons and vector leptoquarks since it is possible to introduce two anomalous couplings $\kappa_{g}$ and $\lambda_{g}$ which are related to the anomalous magnetic and electric quadrupole moments, respectively. We assume here that these quantities are independent in order to work with the most general scenario. The effective Lagrangian describing the interaction of vector leptoquarks $(\Phi)$ with gluons is given by [26]

$$
\begin{aligned}
\mathcal{L}_{V}^{g}= & -\frac{1}{2} V_{\mu \nu}^{i \dagger} V_{i}^{\mu \nu}+M_{\Phi}^{2} \Phi_{\mu}^{i \dagger} \Phi_{i}^{\mu} \\
& -i g_{s}\left[\left(1-\kappa_{g}\right) \Phi_{\mu}^{i \dagger} t_{i j}^{a} \Phi_{\nu}^{j} \mathcal{G}_{a}^{\mu \nu}+\frac{\lambda_{g}}{M_{\Phi}^{2}} V_{\sigma \mu}^{i \dagger} t_{i j}^{a} V_{\nu}^{j \mu} \mathcal{G}_{a}^{\nu \sigma}\right],
\end{aligned}
$$

where there is an implicit sum over all vector leptoquarks, $g_{s}$ denotes the strong coupling constant, $t^{a}$ are the $\mathrm{SU}(3)_{C}$ generators, $M_{\Phi}$ is the leptoquark mass, and $\kappa_{g}$ and $\lambda_{g}$ are the anomalous couplings, assumed to be real. The field strength tensors of the gluon and vector leptoquark fields are, respectively,

$$
\begin{aligned}
\mathcal{G}_{\mu \nu}^{a} & =\partial_{\mu} \mathcal{A}_{\nu}^{a}-\partial_{\nu} \mathcal{A}_{\mu}^{a}+g_{s} f^{a b c} \mathcal{A}_{\mu b} \mathcal{A}_{\nu c}, \\
V_{\mu \nu}^{i} & =D_{\mu}^{i k} \Phi_{\nu k}-D_{\nu}^{i k} \Phi_{\mu k}
\end{aligned}
$$

with the covariant derivative given by

$$
D_{\mu}^{i j}=\partial_{\mu} \delta^{i j}-i g_{s} t_{a}^{i j} \mathcal{A}_{\mu}^{a},
$$

where $\mathcal{A}$ stands for the gluon field.

At present there are no direct bounds on the anomalous parameters $\kappa_{g}$ and $\lambda_{g}$. Here we analyze two scenarios: in the first, called minimal cross section couplings, we minimize the production cross section as a function of these parameters for a given vector leptoquark mass. In the second case, which we name Yang-Mills couplings, we consider that the vector 

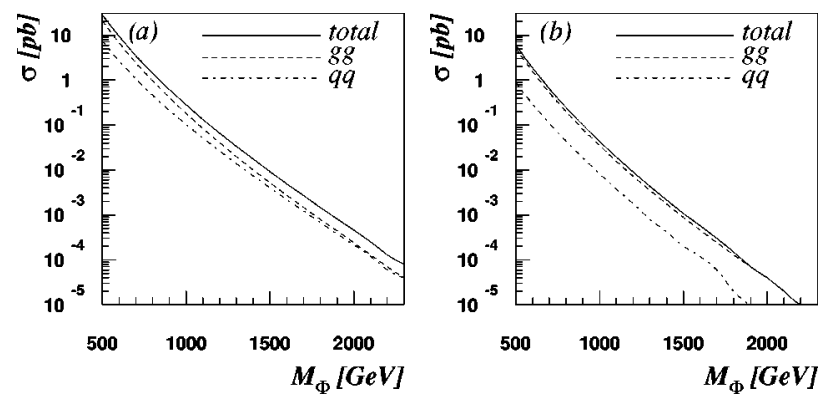

FIG. 1. Production cross sections of vector leptoquarks pairs at the LHC for (a) Yang-Mills coupling and (b) minimum coupling (cross section).

leptoquarks are gauge bosons of an extended gauge group which corresponds to $\kappa_{g}=\lambda_{g}=0$.

\section{SIGNAL SIMULATION AND RATES}

Although the processes for the production of scalar leptoquarks are incorporated in PYTHIA, the vector leptoquark production is absent. In order to study the pair production of vector leptoquarks via the processes (1) and (2) we have created a Monte Carlo generator for these reactions, adding a new external user processes to the PYTHIA 5.7/JETSET 7.4 package [18]. We have included in our simulation two cases of anomalous vector leptoquark couplings to gluons, as well as their decays into fermions.

In our analyses, we assume that the pair production of leptoquarks is due entirely to strong interactions, i.e., we neglect the contributions from $t$-channel lepton exchange via the leptoquark couplings to fermions [26]. This hypothesis is reasonable since the fermionic couplings $g$ and $h$ are bounded to be rather small by the low-energy experiments for leptoquarks masses of the order of TeV's.

The analytical expressions for the scattering amplitudes were taken from the LQPAIR package [27], which was created using the COMPHEP package [28]. The integration over the phase space was done using BASES [29] while we used SPRING for the simulation [29]. An interface between these programs and PYTHIA was specially written.

In our calculations we employed the parton distribution functions CTEQ3L [30], where the scale $Q^{2}$ was taken to be the leptoquark mass squared. Furthermore, the effects of final state radiation, hadronization and string jet fragmentation (by means of JETSET 7.4) have also been taken into account.

The cross sections for the production of vector leptoquark pairs are presented in Fig. 1 for Yang-Mills and minimal couplings. The numerical values of the total cross sections are shown in Table II along with the values of couplings $\kappa_{g}$ and $\lambda_{g}$ that lead to the minimum total cross section. As we can see from this figure, the gluon-gluon fusion mechanism (dashed line) dominates the production of leptoquark pairs for the leptoquark masses relevant for this work at the LHC center-of-mass energy. Moreover, quark-quark fusion is less important in the minimal coupling scenario.

Pairs of leptoquarks decaying into $e^{ \pm}$and a $u$ or $d$ quark produce a pair $e^{+} e^{-}$and two jets as signature. In our analyses we kept track of the $e^{ \pm}$(jet) carrying the largest trans-
TABLE II. Total cross section in pb for the pair production of vector leptoquarks. The values of $\kappa_{g}$ and $\lambda_{g}$ lead to a minimum value of the total cross section for a given leptoquark mass.

\begin{tabular}{cclcc}
\hline \hline & \multirow{2}{*}{ Yang-Mills } & \multicolumn{3}{c}{ Minimal cross section } \\
\cline { 3 - 5 } Mass $(\mathrm{GeV})$ & $\sigma(\mathrm{pb})$ & \multicolumn{1}{c}{$\sigma(\mathrm{pb})$} & $\kappa$ & $\lambda$ \\
\hline 500 & 28.5 & 6.0 & 1.02 & -0.0409 \\
600 & 9.3 & 1.8 & 1.06 & -0.0554 \\
700 & 3.4 & 0.64 & 1.09 & -0.0691 \\
800 & 1.4 & 0.24 & 1.12 & -0.0832 \\
900 & 0.61 & 0.099 & 1.14 & -0.0967 \\
1000 & 0.28 & 0.043 & 1.17 & -0.111 \\
1100 & 0.13 & 0.019 & 1.24 & -0.153 \\
1200 & 0.066 & 0.0091 & 1.24 & -0.163 \\
1300 & 0.033 & 0.0044 & 1.25 & -0.175 \\
1400 & 0.017 & 0.0021 & 1.26 & -0.187 \\
1500 & 0.0092 & 0.0011 & 1.28 & -0.197 \\
1600 & 0.0049 & 0.00056 & 1.29 & -0.208 \\
1700 & 0.0027 & 0.00029 & 1.30 & -0.218 \\
1800 & 0.0014 & 0.00015 & 1.30 & -0.227 \\
1900 & 0.00083 & 0.00008 & 1.31 & -0.238 \\
2000 & 0.00046 & 0.00004 & 1.32 & -0.247 \\
2100 & 0.00026 & 0.00002 & 1.32 & -0.256 \\
\hline \hline
\end{tabular}

verse momentum, which we denoted by $e_{1}\left(j_{1}\right)$, and the $e^{ \pm}$ (jet) with the second largest $p_{T}$, which we called $e_{2}\left(j_{2}\right)$. Furthermore, we mimicked the experimental resolution of the hadronic calorimeter by smearing the final state quark energies according to

$$
\left.\frac{\delta E}{E}\right|_{h a d}=\frac{0.5}{\sqrt{E}} .
$$

The reconstruction of jets was done using the subroutine LUCELL of PYTHIA. The minimum $E_{T}$ threshold for a cell to be considered as a jet initiator has been chosen $2 \mathrm{GeV}$, while we assumed the minimum summed $E_{T}$ for a collection of cells to be accepted as a jet to be $7 \mathrm{GeV}$ inside a cone $\Delta R$ $=\sqrt{\Delta \eta^{2}+\Delta \phi^{2}}=0.7$. The calorimeter was divided on (50 $\times 30)$ cells in $\eta \times \phi$ with these variables in the range $(-5$ $<\eta<5) \times(0<\phi<2 \pi)$.

\section{BACKGROUND PROCESSES AND KINEMATICAL CUTS}

Within the scope of the SM, there are many sources of backgrounds leading to jets accompanied by a $e^{+} e^{-}$pair, which we classify into three classes [17]: QCD processes, electroweak interactions, and top quark production. The reactions included in the QCD class depend exclusively on the strong interaction and the main source of hard $e^{ \pm}$in this case is the semileptonic decay of hadrons possessing quarks $c$ or $b$. The electroweak processes contain the Drell-Yan production of quark pairs and the single and pair productions of electroweak gauge bosons. Because of the large gluon-gluon luminosity at the LHC, the production of top quark pairs is 

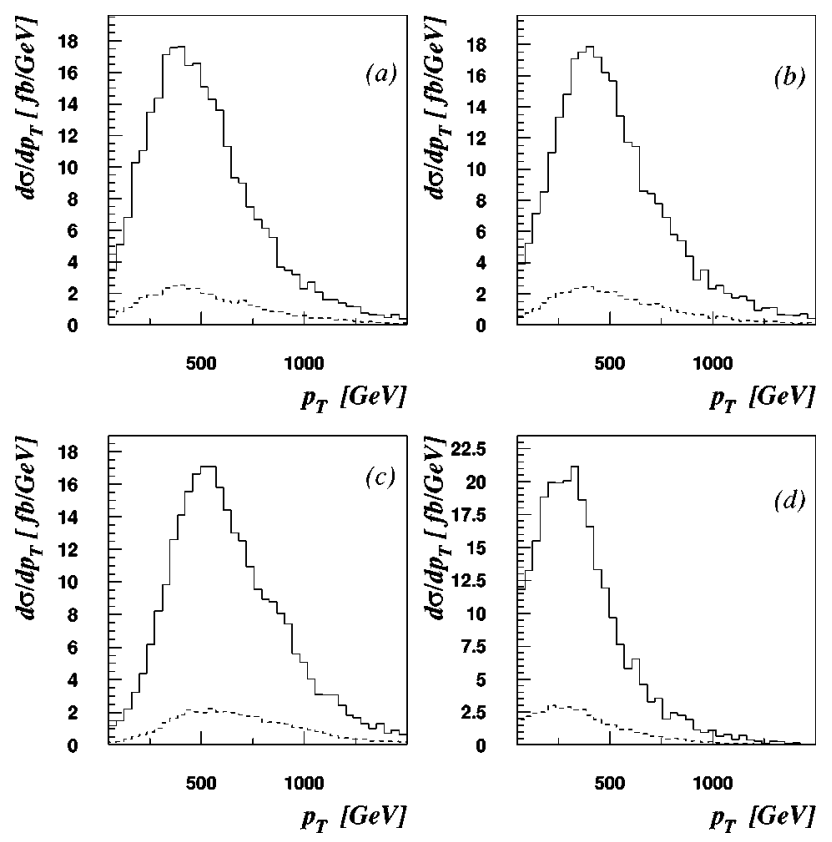

FIG. 2. $p_{T}$ distribution of (a) $e_{1}$, (b) $e_{2}$, (c) $j_{1}$, (d) $j_{2}$ in the pair production of $1 \mathrm{TeV}$ vector leptoquarks with $\beta=1$. The dashed (solid) line stands for the minimum (Yang-Mills) coupling.

important by itself due to its large cross section. These backgrounds have been fully analyzed by us in Ref. [17] and we direct the reader to this reference for further information.

In order to enhance the signal and reduce the SM backgrounds we have devised a number of kinematical cuts in Ref. [17] that we briefly present.

(C1) We require that the leading jets and $e^{ \pm}$be in the pseudorapidity interval $|\eta|<3$.

(C2) The leading leptons $\left(e_{1}\right.$ and $\left.e_{2}\right)$ should have $p_{T}$ $>200 \mathrm{GeV}$.

(C3) We reject events where the invariant mass of the pair $e^{+} e^{-}\left(M_{e_{1} e_{2}}\right)$ is smaller than $190 \mathrm{GeV}$. This cut reduces the backgrounds coming from $Z$ decays into a pair $e^{+} e^{-}$.

(C4) In order to further reduce the $t \bar{t}$ and remaining offshell $Z$ backgrounds, we required that all the invariant masses $M_{e_{i} j_{k}}$ be larger than $200 \mathrm{GeV}$, since pairs $e_{i} j_{k}$ coming from an on-shell top quark decay have invariant masses smaller than $m_{t o p}$. The present experiments are able to search for leptoquarks with masses smaller than $200 \mathrm{GeV}$; therefore, this cut does not introduce any bias on the leptoquark search.

The above cuts reduce to a negligible level all the SM backgrounds [17]. In principle we could also require the $e^{ \pm}$ to be isolated from hadronic activity in order to reduce the QCD backgrounds. Nevertheless, we verified that our results do not change when we introduce typical isolation cuts in addition to any of the above cuts. Since the leptoquark searches at the LHC are free of backgrounds after these cuts [17], the LHC will be able to exclude with 95\% C.L. the regions of parameter space where the number of expected signal events is larger than 3 for a given integrated luminosity.
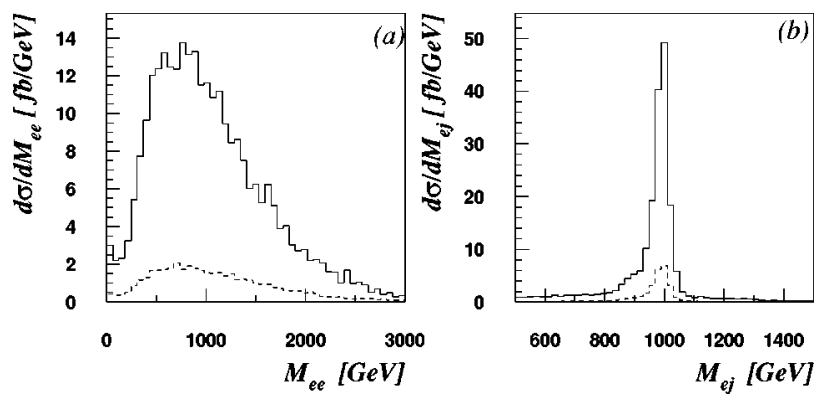

FIG. 3. (a) $e^{+} e^{-}$invariant mass distribution, (b) $e^{ \pm}$-jet invariant mass spectrum adding the four possible combinations. We use the same conventions as Fig. 2.

\section{RESULTS AND CONCLUSIONS}

In order to access the effect of the cuts $(\mathrm{C} 1)-(\mathrm{C} 4)$ we exhibit in Fig. 2 the $p_{T}$ distribution of the two most energetic leptons and jets originating from the decay of a vector leptoquark of $1 \mathrm{TeV}$ for minimal cross section and Yang-Mills couplings to gluons. As we can see from this figure, the $p_{T}$ distributions are peaked at $M_{\Phi} / 2(=500 \mathrm{GeV})$ and also exhibit a large fraction of very hard jets and leptons. The presence of this peak indicates that the two hardest jets and leptons usually originate from the decay of the leptoquark pair. However, we still have to determine which are the lepton and jet coming from the decay of one of the leptoquarks. Moreover, we exhibit in Fig. 3a the $e^{+} e^{-}$invariant mass distribution associated with $1 \mathrm{TeV}$ vector leptoquark events. Clearly the bulk of the $e^{+} e^{-}$pairs are produced at high invariant masses, and consequently the impact of the cut (C3) on the signal is small. Figure $3 \mathrm{~b}$ shows the invariant mass distribution for the four possible $e_{i} j_{k}$ pairs combined in the $1 \mathrm{TeV}$ vector leptoquark case; the cut (C4) does not affect significantly the signal either.

In our analyses of vector leptoquark pair production we applied the cuts $(\mathrm{C} 1)-(\mathrm{C} 4)$ and also required the events to have two $e^{ \pm}$-jet pairs with invariant masses in the range $\left|M_{\Phi} \pm \Delta M\right|$ with $\Delta M$ given in Table III. The pair production cross section after cuts is shown in Fig. 4 for minimal cross section and Yang-Mills couplings. For fixed values of $M_{\Phi}, \kappa_{g}$, and $\lambda_{g}$, the attainable bounds at the LHC on vector leptoquarks depend upon its branching ratio $(\beta)$ into a charged lepton and a jet, which is 0.5 or 1 for the leptoquarks listed in Table I.

We exhibit in Table IV the 95\% C.L. limits on the leptoquark masses that can be obtained from their pair production at the LHC for two different integrated luminosities. In the worse scenario, i.e., minimal cross section couplings, the

TABLE III. Invariant mass bins used in our analyses as a function of the leptoquark mass.

\begin{tabular}{cc}
\hline \hline$M_{\Phi}(\mathrm{GeV})$ & $\Delta M(\mathrm{GeV})$ \\
\hline 500 & 50 \\
1000 & 150 \\
1500 & 200 \\
2000 & 250 \\
\hline
\end{tabular}




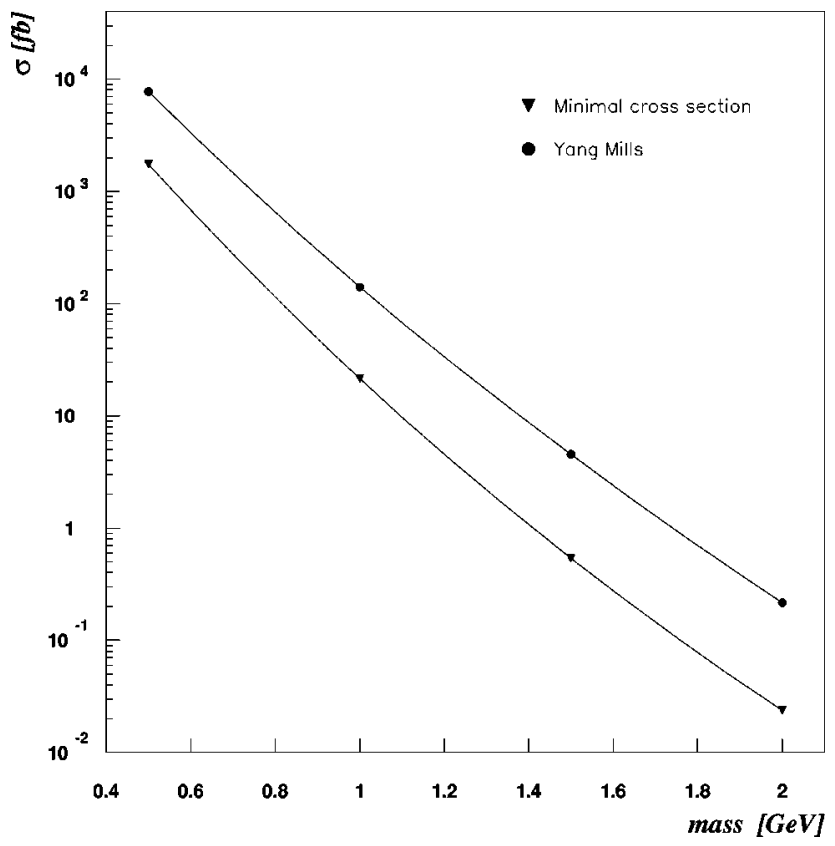

FIG. 4. Cross section after cuts for the production of vector leptoquark pairs assuming Yang-Mills couplings (circles) and minimal cross section couplings (triangles).

LHC will be able to place absolute bounds on vector leptoquark masses smaller than 1.5 (1.6) $\mathrm{TeV}$ for $\beta=0.5$ (1) and an integrated luminosity of $10 \mathrm{fb}^{-1}$. With a larger luminosity of $100 \mathrm{fb}^{-1}$ this bound increases to 1.8 (1.9) TeV. Moreover, the limits are $300 \mathrm{GeV}$ more stringent in the case of YangMills coupling to gluons. At this point it is interesting to compare our results with the ones in Ref. [19]. Requiring
TABLE IV. 95\% C.L. limits on the leptoquark masses that can be obtained from the search for leptoquark pairs for two integrated luminosities $\mathcal{L}=10(100) \mathrm{fb}^{-1}$.

\begin{tabular}{lcc}
\hline \hline & Minimal cross section & Yang-Mills \\
\hline$V_{1 L}$ and $V_{3 L}^{0}$ & $1.5(1.8) \mathrm{TeV}$ & $1.8(2.1) \mathrm{TeV}$ \\
All others & $1.6(1.9) \mathrm{TeV}$ & $1.9(2.3) \mathrm{TeV}$ \\
\hline \hline
\end{tabular}

five signal events like in Ref. [19], we obtain that the LHC will be able to rule out vector leptoquarks with masses smaller than 1.75 (1.86) $\mathrm{TeV}$ for $\beta=0.5$ (1), Yang-Mills couplings, and an integrated luminosity of $10 \mathrm{fb}^{-1}$. Therefore, our cuts are more efficient than the ones proposed in Ref. [19] which lead to a bound of 1.55 (1.65) $\mathrm{TeV}$ in the above conditions.

In brief, the discovery of vector leptoquarks is without any doubt a striking signal of new physics beyond the standard model. The LHC will be of tremendous help in the quest for new physics since, as we have shown, it will be able to discover vector leptoquarks with masses smaller than $1.8-2.3 \mathrm{TeV}$, depending in their couplings to fermions and gluons, through their pair production for an integrated luminosity of $100 \mathrm{fb}^{-1}$.

\section{ACKNOWLEDGMENTS}

This work was partially supported by Conselho Nacional de Desenvolvimento Científico e Tecnológico (CNPq), Fundação de Amparo à Pesquisa do Estado de São Paulo (FAPESP), and by Programa de Apoio a Núcleos de Excelência (PRONEX).
[1] For a review see W. Buchmüller, Acta Phys. Austriaca, Suppl. 27, 517 (1985)

[2] L. Abbott and E. Farhi, Phys. Lett. 101B, 69 (1981); Phys. Lett. B B189, 547 (1981).

[3] See, for instance, P. Langacker, Phys. Rep. 72, 185 (1981).

[4] S. Dimopoulos, Nucl. Phys. B168, 69 (1981); E. Farhi and L. Susskind, Phys. Rev. D 20, 3404 (1979); J. Ellis et al., Nucl. Phys. B182, 529 (1981).

[5] J. L. Hewett and T. G. Rizzo, Phys. Rep. 183, 193 (1989).

[6] ALEPH Collaboration, D. Decamp et al., Phys. Rep. 216, 253 (1992); L3 Collaboration, O. Adriani et al., ibid. 236, 1 (1993); OPAL Collaboration, G. Alexander et al., Phys. Lett. B 263, 123 (1991); DELPHI Collaboration, P. Abreu et al., ibid. 316, 620 (1993); OPAL Collaboration, S. SoldnerRembold, presented at the International Conference on Structure and Interactions of the Photon, Netherlands, 1997, hep-ex/9706003; DELPHI Collaboration, presented at the International Europhysics Conference, Jerusalem, 1994, Report No. DELPHI 97-112 CONF 94.

[7] ZEUS Collaboration, M. Derrick et al., Phys. Lett. B 306, 173 (1993); Z. Phys. C 73, 613 (1997); H1 Collaboration, I. Abt et al., Nucl. Phys. B396, 3 (1993); H1 Collaboration, S. Aid et al., Phys. Lett. B 369, 173 (1996); H1 Collaboration, C. Adloff et al., Z. Phys. C 74, 191 (1997); ZEUS Collaboration, J. Breitweg et al., ibid. 74, 207 (1997).

[8] CDF Collaboration, F. Abe et al., Phys. Rev. D 48, 3939 (1993); Phys. Rev. Lett. 79, 4327 (1997); DØ Collaboration, S. Abachi et al., ibid. 72, 965 (1994); DØ Collaboration, B. Abbott et al., ibid. 79, 4321 (1997); 80, 2051 (1998); CDF Collaboration and DФ Collaboration, C. Grosso-Pilcher et al., hep-ex/9810015.

[9] E. E. Boos et al., DØ Collaboration, DØ Note No. 3416 (http://www-d0.fnal.gov/d0pub/d0_private/3416/ m_VLQDØNote.ps).

[10] O. J. P. Éboli and A. V. Olinto, Phys. Rev. D 38, 3461 (1988); J. L. Hewett and S. Pakvasa, ibid. 37, 3165 (1988); J. Ohnemus et al., Phys. Lett. B 334, 203 (1994); J. Blümlein, E. Boos, and A. Kryukov, Z. Phys. C 76, 137 (1997); M. Krämer et al., Phys. Rev. Lett. 79, 341 (1997); J. L. Hewett and T. Rizzo, Phys. Rev. D 56, 5709 (1997); T. Rizzo, Report No. SLAC-PUB-7284, 1996, hep-ph/9609267; J. E. Cieza Montalvo et al., Phys. Rev. D 58, 095001 (1998); O. J. P. Éboli, R. Z. Funchal, and T. L. Lungov, ibid. 59, 035002 (1998).

[11] W. Buchmüller, R. Rückl, and D. Wyler, Phys. Lett. B 191, 442 (1987). 
[12] J. Wudka, Phys. Lett. 167B, 337 (1986); M. A. Doncheski and J. L. Hewett, Z. Phys. C 56, 209 (1992); J. Blümlein, E. Boos, and A. Pukhov, Mod. Phys. Lett. A 9, 3007 (1994); C. Friberg, E. Norrbin, and T. Sjöstrand, Phys. Lett. B 403, 329 (1997); Z. Kunszt and W. J. Stirling, Z. Phys. C 75, 453 (1997); J. Kalinowski et al., ibid. 74, 595 (1997); J. Blümlein, E. Boos, and A. Krykov, hep-ph/9608483; T. Plehn et al., Z. Phys. C 74, 611 (1997); J. Blümlein, ibid. 74, 605 (1997).

[13] J. L. Hewett and T. G. Rizzo, Phys. Rev. D 36, 3367 (1987); J. L. Hewett and S. Pakvasa, Phys. Lett. B 227, 178 (1987); J. E. Cieza Montalvo and O. J. P. Éboli, Phys. Rev. D 47, 837 (1993); J. Blümein and R. Rückl, Phys. Lett. B 304, 337 (1993); J. Blümlein and E. Boos, Nucl. Phys. B (Proc. Suppl.) 37, 181 (1994); C. G. Papadopoulos, hep-ph/9703372; J. Blümlein, E. Boos, and A. Krykov, Phys. Lett. B 392, 150 (1997); M. S. Berger, hep-ph/9609517; M. A. Doncheski and S. Godfrey, Phys. Lett. B 393, 355 (1998).

[14] F. Cuypers, P. H. Frampton, and R. Rueckl, Phys. Lett. B 390, 221 (1997).

[15] O. J. P. Éboli et al., Phys. Lett. B 311, 147 (1993); H. Nadeau and D. London, Phys. Rev. D 47, 3742 (1993); M. A. Doncheski and S. Godfrey, ibid. 51, 1040 (1995); T. M. Aliev, E. Iltan, and N. K. Pak, ibid. 54, 4263 (1996); F. Cuypers, Nucl. Phys. B474, 57 (1996); M. A. Doncheski and S. Godfrey, Report No. OCIP-C-96-7, 1996, hep-ph/9612385.

[16] G. Bélanger, D. London, and H. Nadeau, Phys. Rev. D 49, 3140 (1994).
[17] O. J. P. Éboli, R. Z. Funchal, and T. L. Lungov, Phys. Rev. D 57, 1715 (1998).

[18] T. Sjöstrand, Comput. Phys. Commun. 82, 74 (1994).

[19] B. Dion, L. Marleau, and G. Simon, Phys. Rev. D 59, 015001 (1999).

[20] O. Shanker, Nucl. Phys. B204, 375 (1982).

[21] W. Buchmüller and D. Wyler, Phys. Lett. B 177, 377 (1986); J. C. Pati and A. Salam, Phys. Rev. D 10, 275 (1974).

[22] M. Leurer, Phys. Rev. Lett. 71, 1324 (1993); Phys. Rev. D 49, 333 (1994).

[23] P. Langacker, Phys. Lett. B 256, 277 (1991); P. Langacker, M. Luo, and A. K. Mann, Rev. Mod. Phys. 64, 87 (1992).

[24] O. J. P. Éboli, M. C. Gonzalez-Garcia, and J. K. Mizukoshi, Nucl. Phys. B443, 20 (1995); Phys. Lett. B 396, 238 (1997).

[25] S. Davidson, D. Bailey, and A. Campbell, Z. Phys. C 61, 613 (1994).

[26] J. Blümlein, E. Boos, and A. Kryukov, Z. Phys. C 76, 137 (1997).

[27] J. Blümlein, E. Boos, and A. Kryukov, program description LQPAIR 1.0 (in preparation).

[28] E. E. Boos et al., Report No. SNUTP-94-116, hep-ph/9503280; E. E. Boos et al., in Proceedings of the Xth International Workshop on High Energy Physics and Quantum Field Theory, Moscow, 1995, edited by B. Levtchenko and V. Savrin, Report No. QFTHEP-95; V. A. Ilyin, D. N. Kovalenko, and A. E. Pukhov, INP MSU Report No. 95-2/366.

[29] S. Kawabata, Comput. Phys. Commun. 41, 127 (1996).

[30] H. L. Lai et al., Phys. Rev. D 51, 4763 (1995). 